\title{
Reports of clinical trials: Ethical aspects
}

Robert M. Sade, MD

See related articles on pages $229,233,241,243,247$, and 249.
From the Department of Surgery and the Institute of Human Values in Health Care, Medical University of South Carolina, Charleston, SC.

Received for publication Oct 25, 2005; accepted for publication Mar 3, 2006.

Address for reprints: Robert M. Sade, MD, Medical University of South Carolina, Department of Surgery, 96 Jonathan Lucas St, Suite 409, PO Box 250612, Charleston, SC 29425 (E-mail: sader@musc.edu).

J Thorac Cardiovasc Surg 2006;132:245-6

$0022-5223 / \$ 32.00$

Copyright () 2006 by The American Association for Thoracic Surgery

doi:10.1016/j.jtcvs.2006.03.042
$\mathrm{T}$

Tiruvoipati and colleagues ${ }^{1}$ have shown us glaring deficiencies in the reporting of randomized controlled trials (RCTs) in the cardiothoracic surgery (CTS) literature. The CONSORT statement, a checklist and flowchart used in writing reports of RCTs, is a tool that can be used to improve RCT reports; it is endorsed by most major medical journals but by none of the major cardiothoracic journals. Why is this? One reason might be that RCTs are not as applicable to surgical as to medical disciplines. Indeed, the authors observe that RCTs are only half as prevalent in our journals as they are in general medical journals, and therefore CTS journal editors might be less inclined to pay attention to quality of reporting of low-incidence articles.

RCTs, the gold standard of clinical investigation, are less common in CTS journals than in medical journals for several reasons. Surgical clinical trial protocols that use no-treatment or placebo control groups are often not ethical, not desirable, or both. Double blinding is usually not practicable because the surgeon must know what he or she is doing (although blinding of assessors and analysts can be done). Moreover, groups of patients in need of specific operations are often small and not easily amenable to statistical analysis. Of greatest importance, however, is a special characteristic of surgical procedures: they are associated with a learning curve and small incremental improvements in technique that progressively improve outcomes. ${ }^{2}$ Few of these limitations apply to drug trials and other medical investigations, and therefore we should not find it surprising that RCTs are more frequent in medical than in surgical journals.

Nevertheless, Tiruvoipati and colleagues ${ }^{1}$ identified 62 RCTs in CTS journals in 2003. This is not a trivial number, and therefore perhaps it is time for CTS journals to look more closely at the quality of the reports they publish. Accurate and complete reporting of relevant information arising from investigations benefits everyone: physicians-investigators because they learn what will benefit their patients and what will not; the human patients-subjects because of fulfillment of their expectations that serving as experimental subjects will advance health care and perhaps directly benefit themselves and that the risks they have taken have not been wasted; and future patients because they will be the beneficiaries of new information about drugs, methods, or operations. Conversely, inaccurate and incomplete reporting of clinical investigations harms those same individuals and groups.

Reporting of clinical investigations can be compromised in many ways. For example, industrial sponsors might erect barriers to publication of unfavorable results; investigators might design, execute, or analyze results of studies poorly or report results selectively; and journal reviewers and editors might overlook errors and deficiencies. Therefore the critical need for accurate and complete reporting of clinical investigations places certain burdens on individuals and institutions:

- sponsors must allow investigators freedom to report without bias or undue influence whatever the investigator believes is relevant, whether positive or negative and whether beneficial or harmful to product marketing;

- investigators must analyze their results objectively and report all relevant information, including conclusions and recommendations that are justified by the data, without overreaching and without understating;

- peer reviewers should evaluate the clarity and completeness of the report and judge whether the conclusions and recommendations are justified by the data reported; and 
- editors and editorial boards should use mechanisms to ascertain freedom of investigators in reporting and interpreting their data and encourage complete and accurate reporting through use of tools such as the CONSORT statement.

Forms of investigation other than RCTs might provide reliable and useful new knowledge in CTS, such as prospective uncontrolled clinical trials, retrospective examination of groups of patients or operations, and analysis of large databases, such as the Society of Thoracic Surgeons' National Database. ${ }^{3}$ The authors of a recent study of RCTs in the CTS literature commented that "for most study questions in clinical surgery, comparative analysis of large case series and databases will provide more robust evidence" than RCTs. ${ }^{4}$ Like RCTs, such investigations might be reported well or poorly, and it might be wise for CTS journal editors to consider developing and requiring adherence to checklists (perhaps based on CONSORT) for those reports as well. Certainly, however, the data reported by Tiruvoipati and col- leagues ${ }^{1}$ make it incumbent on editors and editorial boards to assess critically their own practices regarding reporting of RCTs and other human subject research and to consider adopting the CONSORT statement for reporting RCTs. They should make any changes in their policies that seem likely to improve reporting of clinical investigations.

\section{References}

1. Tiruvoipati R, Balasubramanian SP, Atturu G, Peek GJ, Elbourne D. Improving the quality of reporting randomized controlled trials in cardiothoracic surgery: the way forward. J Thorac Cardiovasc Surg. 2006;132:233-40.

2. Morreim EH. Surgically implanted devices: ethical challenges in a very different kind of research. Thorac Surg Clin. 2005;15:555-63.

3. STS National Database. Available at: http://www.sts.org/sections/ stsnationaldatabase/. Accessed October 18, 2005.

4. Anyanwu AC, Treasure T. Surgical research revisited: clinical trials in the cardiothoracic surgical literature. Eur J Cardiothorac Surg. 2004; 25:299-303 